\title{
Performance of Multipoint Relaying in Ad Hoc Mobile Routing Protocols
}

\author{
Philippe Jacquet, Anis Laouiti, Pascale Minet, and Laurent Viennot \\ INRIA \\ Domaine de Voluceau \\ 78153 Le Chesnay cedex \\ France \\ \{philippe.jacquet, anis.laouiti, pascale.minet, laurent.viennot\}@inria.fr
}

\begin{abstract}
We analyze the performance of ad hoc pro-active routing protocols. In particular we focuse on the multipoint relay concept introduced in OLSR protocol and which brings a significant improvement in broadcast control traffic overhead.We analyze the performance in two radio network model: the random graph model and the unit graph model. The random graph is more suitable for the modelization of indoor networks. The unit graph is more suitable for outdoor networks. We compare the performance of OLSR with the performance of basic link state protocols using full flooding.
\end{abstract}

\section{Introduction}

Radio networking is emerging as one of the most promising challenge made possible by new technology trends. Mobile Wireless networking brings a new dimension of freedom in internet connectivity. Among the numerous architectures that can be adapted to radio networks, the $A d$ hoc topology is the most attractive since it consists to connect mobile nodes without pre-existing infrastructure. When some nodes are not directly in range of each other there is a need of packet relaying by intermediate nodes. The working group MANet of Internet Engineering Task Force (IETF) is standardizing routing protocol for ad hoc wireless networking under Internet Protocol (IP). In MANet every node is a potential router for other nodes. The task of specifying a routing protocol for a mobile wireless network is not a trivial one. The main problem encounterd in mobile networking is the limited bandwidth and the high rate of topological changes and link failure caused by node movement. In this case the classical routing protocol as Routing Internet Protocol (RIP) and Open Shortest Path First (OSPF) first introduced in ARPANET [1] are not adapted since they generate too much control traffic and can only accept few topology changes per minute.

MANet working group proposes two kinds of routing protocols: the reactive protocols and the pro-active protocols. The reactive protocols such as AODV [3], DSR [2, and TORA [4], do not need control exchange data in absence of data traffic. Route discovery procedure is invoked on demand when a source has a new connection pending toward a new destination. The route discovery procedure in 
general consists into the flooding of a query packet and the return of the route by the destination. The exhaustive flooding can be very expensive, thus creating delays in route establishment. Furthermore the route discovery via flooding does not guarantee to create optimal routes in terms of hop-distance.

The pro-active protocols such as Optimized Link State Routing (OLSR) [5], TBRPF [6], need periodic update with control packet and therefore generates an extra traffic which adds to the actual data traffic. The control traffic is broadcasted all over the network via optimized flooding. Optimized flooding is possible since nodes permanently monitor the topology of the network. OLSR uses multipoint relay flooding which very significantly reduce the cost of such broadcasts. Furthermore, the node have permanent dynamic database which make optimal routes immediately available on demand. The protocol OLSR has been adapted from the intra-forwarding protocol in HIPERLAN type 1 standard [7. Most of the salient features of OLSR such as multipoint relays and link state routing are already existing in the HIPERLAN standard.

The aim of the present paper is to analyze the performance of the multipoint relaying concept of OLSR under two models of network: the random graph model and the unit graph model. The paper is divided into four main sections. The use of analytical models is very interesting because it captures the essential of the algorithms that cannot be captured by simulations because of the combinatorics explosion of parameters to tune. This follows the first attempts of ad hoc routing analytical modeling in [15,16]. The first section summarizes the main feature of OLSR protocol. The second section introduces and discusses the graph models. The third section develops the performance analysis of OLSR with respect to the graph models. We give few theoretical theorems about the performance of multipoint relaying without proof due to the lack of place but with due reference to reports and article containing in extenso the proofs (mostly in the research report [14]).

\section{The Optimized Link State Routing Protocol}

\subsection{Non Optimized Link State Algorithm}

Before introducing the optimized link state routing we make a brief reminder about non optmized link state such as OSPF. In an ad hoc network, we call link, a pair of two nodes which can hear each other. In order to achieve unicast transmission, it is important here to use bidirectionnal link (IEEE 802.11 radio LAN standard requires a two way packet transmission). However due to sensitivity of power discrepancies, unidirectional links can arise in the network. The use of unidirectional links is possible but require different protocols and is omitted here. Each link in the graph is a potential hop for routing packets. The aim of a link state protocol is that each node has sufficient knowledge about the existing link in the network in order to compute the shortest path to any remote node.

Each node operating in a link state protocol performs the two following tasks:

- Neighbor discovery: to detect the adjacent links;

- Topology broadcast: to advertize in the whole network about important adjacent links. 
By important adjacent links we mean a subset of adjacent links that permit the computation of the shortest path to any destination.

The simplest neighbor discovery consists for each node to periodically broadcast full hello packets. Each full hello packet contains the list of the heared neighbor by the node. The transmission of hello packets is limited to one hop. By comparing the list of heared nodes each host determine the set of adjacent bidirectional links.

A non optimized link state algorithm performs topology broadcast simply by periodically flooding the whole network with a topology control packet containing the list of all its neighbor nodes (i.e. the heads of its adjacent links). In other words, all adjacent links of a node are important. By flooding we mean that every node in the network re-broadcast the topology control packet upon reception. Using sequence number prevents the topology control packet to be retransmitted several times by the same node. The number of transmissions of a topology control packet is exactly $N$, when $N$ is the total number of in the network, and when retransmission and packet reception are error free.

If $h$ is the rate of hello transmission per node and $\tau$ the rate of topology control generation, then the actual control overhead in terms of packet transmitted of OSPF is

$$
h N+\tau N^{2}
$$

In terms of bytes transmitted, more precisely in IP addresses unit, the overhead is

$$
h N M+\tau N^{2} M
$$

where $M$ is the average number of adjacent links per node. If $M$ is of the same order than $N$ then the overhead is cubic in $N$. Notice that the topology broadcast overhead is one order of magnitude larger than the neighbor discovery overhead.

Notice that for non-optimized link state routing the hello and topology control packet can be the same.

\subsection{OLSR and MultiPoint Relay Nodes}

The Optimized Link State Routing protocol is a link state protocol which optimizes the control overhead via two means:

1. the important adjacent links are limited to MPR nodes;

2. the flooding of topology control packet is limited to MPR nodes (MPR flooding).

The concept of MultiPoint Relay (MPR) nodes has been introduced in [7. By MPR set we mean a subset of the neighbor nodes of a host which covers the two-hop neighborhood of the host. The smallest will be the MPR set the more efficient will be the optimization. We give a more precise definition of the multipoint relay set of a given node $A$ in the graph. We define the neighborhood of $A$ as the set of nodes which have an adjacent link to $A$. We define the twohop neighborhood of $A$ as the set of nodes which have an invalid link to $A$ but 
have a valid link to the neighborhood of $A$. This information about two-hop neighborhood and two-hop links are made available in hello packets, since every neighbor of $A$ periodically broadcasts their adjacent links. The multipoint relay set of $A(\operatorname{MPR}(A))$ is a subset of the neighborhood of $A$ which satisfies the following condition: every node in the two-hop neighborhood of $A$ must have a valid link toward $\operatorname{MPR}(A)$.

The smaller is the Multipoint Relay set is, the more optimal is the routing protocol. [13 gives an analysis and examples about multipoint relay search algorithms. The MPR flooding can be used for any kind of long hole broadcast transmission and follows the following rule:

A node retransmits a broadcast packet only if it has received its first copy

from a node for which it is a multipoint relay.

Reference [7] gives a proof that such flooding protocol (selective flooding) eventually reaches all destinations in the graph. 7] also gives a proof that for each destination in the network, the subgraph made of all MPR links in the network and all adjacent links to host $A$ contains a shortest path with respect to the original graph.

Therefore the multipoint relays improve routing performance in two aspects: first it significantly reduces the number of retransmissions in a flooding or broadcast procedure; second it reduces the size of the control packets since OLSR nodes only broadcast its multipoint relay list instead of its whole neighborhood list in a plain link state routing algorithm.

In other words if $D_{N}$ is the average number of MPR links per node and $R_{N}$ the average number of retransmission in an MPR flooding, then the control traffic of OLSR is, in packet transmitted:

$$
h N+\tau R_{N} N,
$$

and, in IP addresses transmitted:

$$
h M N+\tau R_{N} D_{N} N .
$$

Notice that when the nodes selects all their adjacent links as MPR links, we have $D_{N}=M$ and $R_{N}=N$ : we have the overhead of a full link state algorithm. However we will show that straightforward optimizations make $D_{N} \ll M$ and $R_{N} \ll N$ gaining several orders of magnitude in topology broadcast overhead. Notice that the neighbor discovery overhead is unchanged. Summing both overhead we may expect that OLSR has an overhead reduced of an magnitude order with respect to full link state protocol.

The protocol as it is proposed in IETF may differ to some details from this very simple presentation. The reason is for second order optimization with regards to mobility for example. For example hosts in actual OLSR do not advertize their MPR set but their MPR selector set, i.e. the subset of neighbor nodes which have selected this host as MPR.

\subsection{MPR Selection}

Finding the optimal MPR set is an NP problem as proven in [8]. However there are very efficient heuristics. Amir Qayyum [13] has proposed the following one: 
1. select as MPR, the neighbor node which has the largest number of links in the two-hop neighbor set;

2. remove this MPR node from the neighbor set and the neighbor nodes of this MPR node from the two-hop neighbor set;

3. the previous steps until the two-hop neighbor set is empty.

An ultimate refinement is a prior operation which consists into detecting in the two-hop neighbor the node which have a single parent in the neighbor set. These parents are selected as MPR and are eliminated from the neighbor set, and their neighbor are eliminated in the two-hop neighbor set.

It is proven in 810 that this heuristic is optimal by a factor $\log M$ where $M$ is the size of the neighbor set (i.e. the heuristical MPR set is at most $\log M$ times larger than the optimal MPR set).

\section{The Graph Models}

The modelization of ad hoc mobile network is not an easy task. Indeed the versatility of radio propagation in presence of obstacles, distance attenuation and mobility is the source of incommensurable difficulties. In passing one should notice that mobility not only encompass host mobility but also the mobility of the propagation medium. For example when a door is open in a building, then the distribution of links change. If a truck passes between two host it may switch down the link between them. In this perspective building a realistic model that is tractable by analysis is hopeless. Therefore we will focuse to build models dedicated to specific scenarios.

There are two kinds of scenarios: the indoor scenarios and the outdoor scenarios. For the indoor scenario we will use the random graph model. For the outdoor scenarios we will use the random cartesian graph model. The most realistic model lies somewhere between the random graph model and the random cartesian graph model.

\subsection{The Random Graph Model for Indoor Networks}

In the following we consider a wireless indoor network made of $N$ nodes. The links are distributed according to a random graph with $N$ vertices an link probability $p$. In other words, a link exists between two given nodes with probability $p$. Link's existence are independent from one pair of nodes to another. Figure 1 shows an example of a random graph with $(N, p)=(10,0.7)$, the nodes have been drawed in concentric mode just for convenience.

The random graph model implicitly acknowledge the fact that in an indoor network, the main cause of link obstruction is the existence of random obstacle (wall, furniture) between any pair of nodes. The fact that the links are independently distributed between node pairs assumes that these obstacles are independly distributed with respect to node position, which of course is never completely true. However the random graph model is the simplest satisfactory model of indoor radio network and provides excellent results as a starting point. 


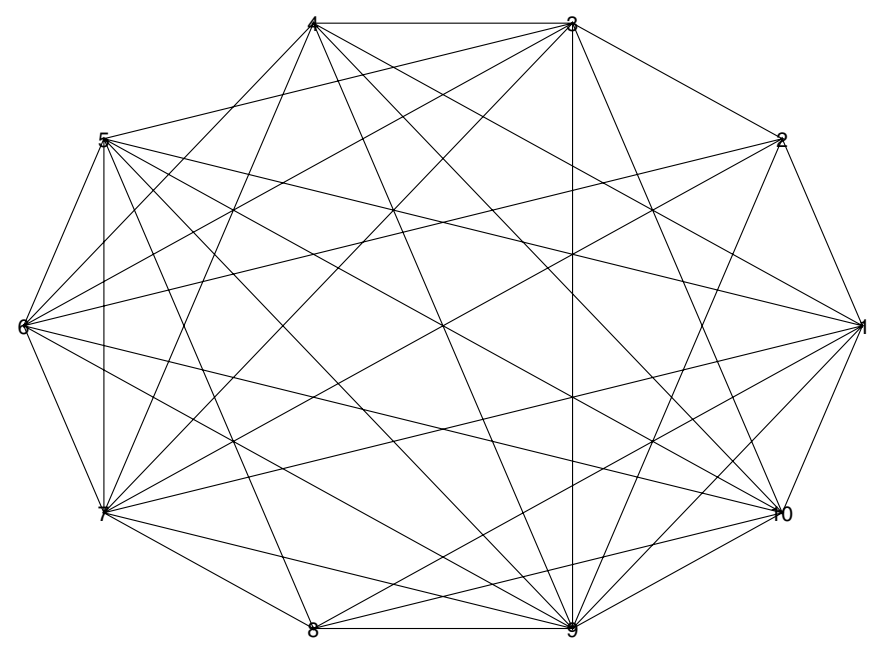

Fig. 1. A random graph with $n=10$ and $p=0.7$, generated by Maple

When the network is static, then the graph does not change during the time. It is clear that nodes does not frequently change position in indoor model, but the propagation medium can vary. In this case the random graph may vary with the time. One easy way to model time variation is to assume random and independent link lifetime. For example, one can define $\mu$ as link variation rate, i.e. the rate at which each link may come down or up. During an interval $[t, t+d t]$ a link can change its status with probability $\mu d t$, i.e. it takes status "up" or "down" with probability $p$, independently of its previous status. The effect of mobility won't be investigated in the present paper.

\subsection{The Random Unit Graph Model for Outdoors Networks}

To explain this kind of graph it suffices to refer to a very simple example. Let $L$ be a non-negative number and let us define a two-dimensional square of size $L \times L$ unit lengths. Let consider $N$ nodes uniformly distributed on this square. The unit graph is the graph obtained by systematically linking pairs nodes when their distance is smaller or equal to the unit length. This model of graph is well adapted to outdoor networks where the main cause of link failure is the attenuation of signal by the distance. In this case the area where a link can be established with a given host is exactly the disk of radius the radio range centered on the host. However the presence of obstacle may give a more twisted shape to the reception area (that may not be single connected).

Figure 2 shows a random unit graph of dimension two. The random unit graph is built in two step: the first step is the uniform distribution of the point on the rectangle area; the second step is the link distribution between node pair according to their distance. 


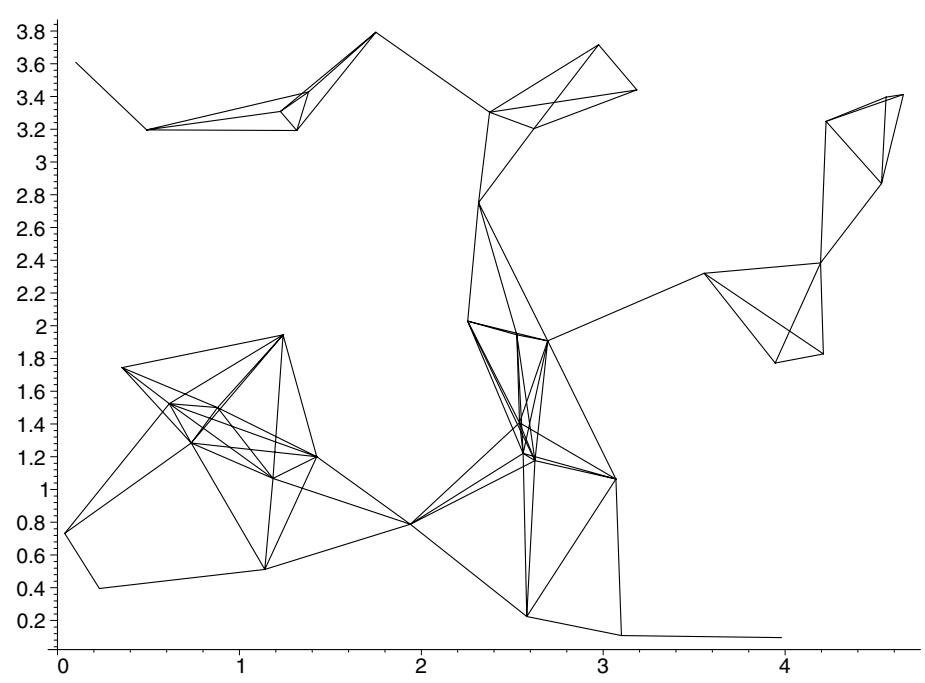

Fig. 2. The random Unit graph derived from random forty points locations in a $5 \times 4$ rectangle

The reception area may also change with the time, due to node mobility, obstacle mobility, noise or actual data traffic. In the present paper we will assume that the network is static.

Of course, the unit graph can be defined on other space than the plane. For example a unit graph can be defined on a 1D segment, modeling a mobile network made of cars on a road. It can be a cube in the air, modeling a mobile network made of airplanes, for example.

\section{Analysis of OLSR in the Random Graph Model}

The proof of the results listed in this section can be found in [9].

Most pro-active protocols (like OLSR) have the advantage to deliver optimal routes (in term of hop number) to data transfers (the proof of this is in 7 and [14]). The analysis of optimal routes is very easy in random graph models since a random graph tends to be of diameter 2 when $N$ tends to infinity with fixed $p$.

Theorem 1. The optimal route between two random nodes in a random graph, when $N$ tends to infinity, is either of length 1 with probability p,or of length 2 with probability $q=1-p$.

Theorem 2. For all $\varepsilon>0$, the optimal MPR set size $D_{N}$ of any arbitrary node is smaller than $(1+\varepsilon) \frac{\log N}{-\log q}$ with probability tending to 1 when $N$ tends to infinity. 
Notice that $D_{N}=O(\log N)$ which very favorably compares to the size of the the whole host neighborhood which (in average $p N$ ) and considerably reduces the topology broadcast.

Theorem 3. The broadcast or flooding via multipoint relays takes in average a number $R_{N}$ of retransmissions smaller than $(1+\varepsilon) \frac{\log N}{-p \log q}$.

Corollary 1. The cost of OLSR control traffic for topology broadcast in the random graph model is $O\left(N(\log N)^{2}\right)$ compared to $O\left(N^{3}\right)$ with plain link state algorithm.

Remark: Notice that the neighbor sensing in $O\left(N^{2}\right)$ is now the dominant source of control traffic overhead.

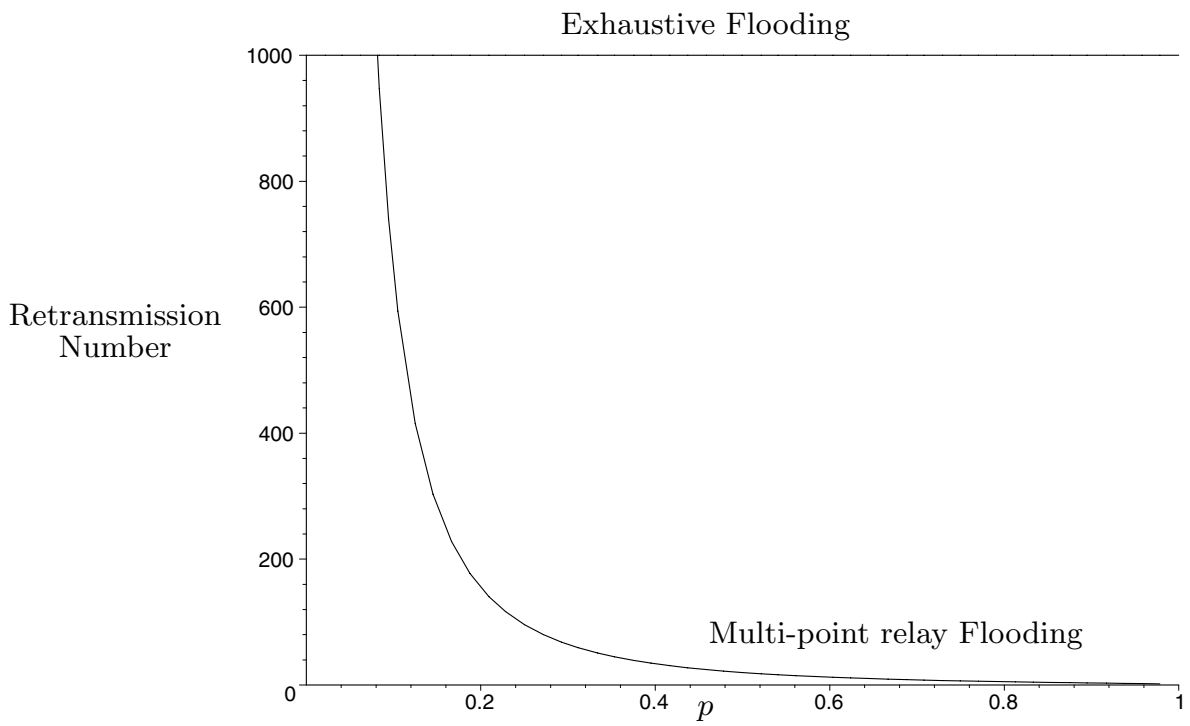

Fig. 3. average number of retransmissions in multi-point relay flooding with $N=1000$ and $p$ variable

\section{Analysis of OLSR in the Random Unit Graph}

\subsection{Results in 1D and 2D Random Unit Graphs}

We present results for $1 \mathrm{D}$ and $2 \mathrm{D}$ random unit graphs. The proof of the results shown in this section can be found in [14. A 1D Unit graph can be made of $N$ nodes uniformly distributed on a strip of land whose width is smaller than the radio range (set as unit length). We assume that the length of the land strip is $L$ unit length. 
Theorem 4. The size of the MPR set $D_{N}$ of a given host is 1 when the host is at less than one radio hop to the end to one end of the strip, and 2 otherwise.

Theorem 5. The MPR flooding of a broadcast message originated by a random node takes $R_{N}=\lfloor L\rfloor$ retransmission of the message when $N$ tends to infinity and $L$ is fixed.

Notice this is assuming an error free retransmission. In case of error, the retransmission stops at the first MPR which does not receive correctly the message. In order to cope with this problem one may have to add redundance in the MPR set which might be too small with regard to this problem.

Notice that these figures favorably compare with plain link state where $D_{N}=$ $M=N / L$ and $R_{N}=N$.

The analysis in $2 \mathrm{D}$ is more interesting because it gives less trivial results.

Theorem 6. When $L$ is fixed and $N$ increases, then the average size of the $M P R$ set, $D_{N}$ tends to be smaller than $3 \pi\left(N /\left(3 L^{2}\right)\right)^{1 / 3}=3 \pi(M /(3 \pi))^{1 / 3}$.

Notice that this figure compares favorably with plain link state where $D_{N}=$ $M=N / L^{2}$.

Figure 4 displays simulation results for dimension 2. The heuristic has been applied to the central node of a random $4 \times 4$ unit graph. The convergence in $M^{1 / 3}$ is clearly shown. Notice that in this very case the upper bound of $D_{N}$ is at least greater by a factor 2 than actual values obtained by simulations. Figure 5 summarizes the results obtained for quantity $D_{N}$ in the random graph model for dimension 1 and 2 . The results for dimension 2 have been simulated.

Theorem 7. The MPR flooding of a broadcast message originated by a random node takes $R_{N}=O\left(\left(N L^{4}\right)^{1 / 3}\right)$ retransmissions of the message when $N$ tends to infinity and $L$ is fixed.

\subsection{Comparison with Dominating Set Flooding}

In 11 Wu and Li introduced the concept of dominating set. They introduced two kinds of dominating set that we will call, the rule 1 dominating set and the rule 2 dominating set. In this section we establish quantative comparisons between the performance of dominating set flooding and MPR flooding. In particular we will show that dominating set floodings does not outperform significantly full flooding in random graph models and in random unit graph of dimension 2 and higher. Rule 1 dominating set does not outperform significantly full flooding in random unit graph model of dimension 1. MPR flooding outperforms both dominating set flooding in any graph models studied in this paper.

The dominating set flooding consists into restricting the retransmission of a broadcast message to a subset of nodes, called the dominating set. Rule 1 and rule 2 consist into two different rules of dominating set selection. The rules consist into compairing neighbor sets (for example by checking hellos). For a node $A$ we denote by $\mathcal{N}(A)$, the neighbor set of node $A$.

In rule 1 , a node $A$ does not belong to the dominating if and only if there exists a neighbor $B$ of $A$ such that 


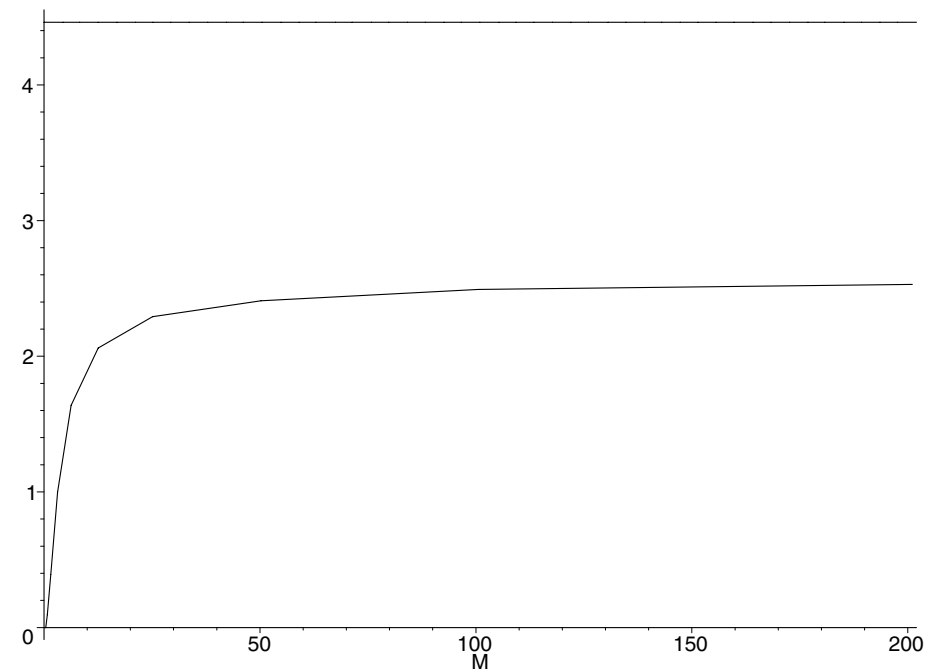

Fig. 4. Bottom: simulated quantity $D_{N} / M^{1 / 3}$ versus the number of neighbor $M$ for the central position in a $4 \times 4$ random unit graph,top: upper bound obtained in theorems.

full link state

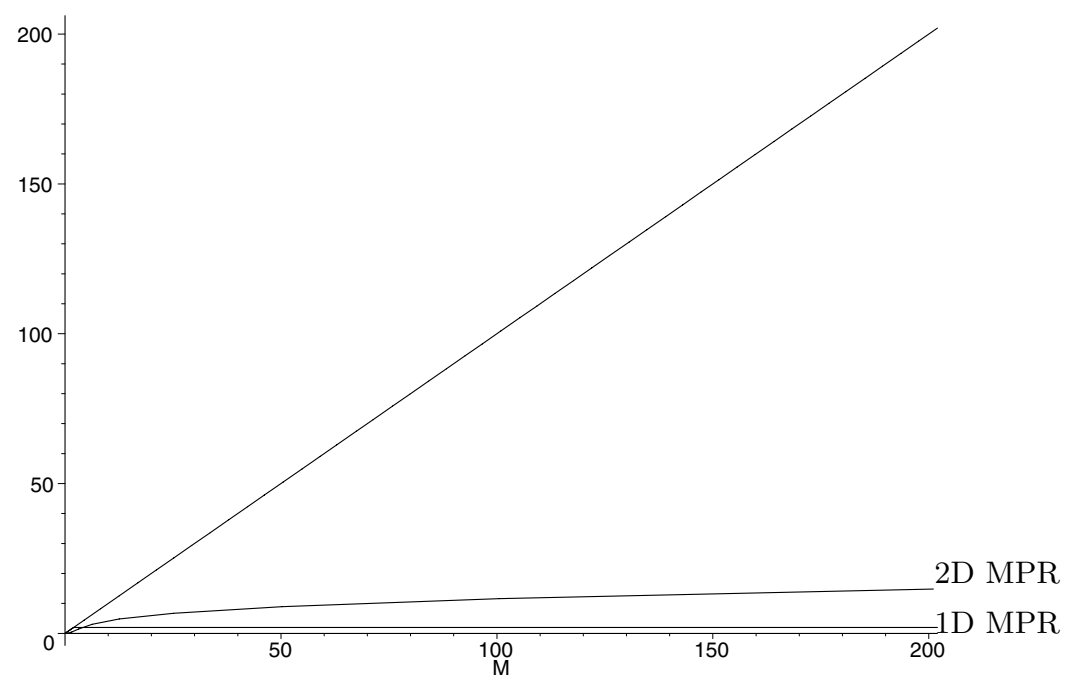

Fig. 5. Unit graph model from bottom to top, average number of MPR for 1D, 2D and full links state protocol versus the average number of neighbor nodes $M$.

1. $B$ is in the dominating set;

2. the IP address of $B$ is higher than the IP address of $A$;

3. $\mathcal{N}(A) \subset \mathcal{N}(B)$.

In this case one says that $B$ dominates $A$ in rule 1. 
In rule 2 , a node $A$ does not belong to the dominating if and only if there exist two neighbor $B$ and $C$ of $A$ such that

1. $B$ and $C$ are in the dominating set;

2. nodes $B$ and $C$ are neighbors;

3. the IP addresses of $B$ and $C$ are both higher than the IP address of $A$;

4. $\mathcal{N}(A) \subset \mathcal{N}(B) \cup \mathcal{N}(C)$.

In this case one says that $(B, C)$ dominates $A$ in rule 2 .

We first, look at the performance of dominating set flooding in the random graph model $(N, p)$.

Theorem 8. The probability that a node in a random graph $(N, p)$ does not belong to the dominating set is smaller than $N(1-(1-p) p)^{N}$ in rule 1 , and smaller than $N^{2}\left(1-(1-p)^{2} p\right)^{N}$ in rule 2.

Theorem 9. In the random unit graph model of dimension 1, assuming independence between node location and node IP addresses, the probability that a node does not belong to the dominating set in rule 1 is smaller than $\frac{4}{M}$ and the average size of the dominating set in rule 2 is $\max \{0,2 L-1\}$.

Remark: The proofs of these theorem can be found in [14]. The density of the dominating set in rule 2 is twice than the density of retransmitters in MPR flooding when the network model is the random unit graph of dimension one.

In random graph of dimension 2 and higher the probabilities that a node does not belong to the dominating set in rule 1 or in rule 2 are $O(1 / M)$ since it is impossible to cover one unit disk with two unit disk that have different centers.

\section{Conclusion and Further Works}

We have presented a performance evaluation of OLSR mobile ad-hoc routing protocols in the random graph model and in the random unit graph model. The originality of the performance evaluation is that it is completely based on analytical methods (generating function, asymptotic expansion) and does not rely on simulation software. The random graph model is enough realistic for indoor or short range outdoor networks where link fading mainly comes from random obstacles. The random unit graph model is realistic for long range outdoor networks where link fading mainly comes from distance attenuation. In this case the random graph model can be improved by letting the parameter $p$ depending on distance $x$ between the nodes. This will be subject of further works.

\section{References}

1. J.M. McQuillan, I. Richer, E.C. Rosen, "The new routing algorithm for the ARPANET," IEEE Trans. Commun. COM-28:711-719. 
2. D.B. Johnson, D.A. Maltz, "Dynamic Source Routing in Ad Hoc Wireless Networks," in Mobile Computing, Ch. 5, pp 153-181, Kluwer Academic Publisher, 1996.

3. C.E. Perkins, E.M. Royer, "Ad Hoc On-Demand Distance Vector Routing," IEEE Workshop on Mobile Computing Systems and Applications, pp. 90-100, 1999.

4. M.S. Corson, V. Park, "Temporallly ordered routing algorithm," draft-ietf-manettora-spec-02.txt, 1999.

5. P. Jacquet, P. Muhlethaler, A. Qayyum, A. Laouiti, L. Viennot, T. Clausen, MANET draft "draft-ietf-manet-olsr-02.txt," 2000.

6. B. Bellur, R. Ogier, F. Templin, "Topology broadcast based on reverse-path forwarding," draft-ietf-manet-tbrpf-01.txt, 2001.

7. P. Jacquet, P. Minet, P. Muhlethaler, N. Rivierre, "Increasing reliability in cablefree Radio LANs: Low level forwarding in HIPERLAN," in Wireless Personal Communications Vol 4, No 1, pp. 51-63, 1997.

8. L. Viennot, "Complexity results on election of multipoint relays in wireless networks," INRIA RR-3584, 1998. http://www.inria.fr/rrrt/rr-3584.html

9. P. Jacquet, A. Laouiti, "Analysis of mobile ad hoc network routing protocols in random graphs," INRIA RR-3835, 1999. http://www.inria.fr/rrrt/rr-3835.html

10. A. Qayyum, L. Viennot, A. Laouiti, "Multipoint relaying: An efficient technique for flooding in mobile wireless networks," INRIA RR-3898, 2000. http://www.inria.fr/rrrt/rr-3898.html

11. J. Wu, H. Li, "On calculating connected dominating set for efficient routing in ad hoc wireless networks," in Proc. DIAL M, 1999.

12. P. Jacquet, L. Viennot, "Overhead in mobile ad hoc network protocols," INRIA RR-3965, 2000.http://www.inria.fr/rrrt/rr-3965.html

13. A. Qayyum, Analysis and evaluation of channel access schemes and routing protocols for wireless networks, Thèse de l'Université Paris 11, 2000.

14. P. Jacquet, A. Laouiti, P. Minet, L. Viennot, "Performance analysis of OLSR multipoint relay flooding in two ad hoc wireless network models," INRIA Research Repport RR-4260, 2001. http://www.inria.fr/rrrt/rr-4260.html

15. A. D. Aron et al., "Analytical comparison of local and end-to-end error recovery in reactive routing protocols for MANET," 3rd ACM MSWiM 2000.

16. A. Boukerche et al., "Analysis of randomized congestion control with DSDV routing in ad hoc wireless networks," in JPDC, pp. 967-995, Vo 61, 2001. 